\title{
Two cases of verified clinical failures using internationally recommended first-line cefixime for gonorrhoea treatment, Norway, 2010
}

M Unemo (magnus.unemo@orebroll.se) ${ }^{1}$, D Golparian¹, G Syversen², D F Vestrheim ${ }^{3,4}$, H Moi3,5

1. Swedish Reference Laboratory for Pathogenic Neisseria, Department of Laboratory Medicine, Microbiology, Örebro University Hospital, Örebro, Sweden

2. Department of Microbiology, Oslo University Hospital, Ullevål, Oslo, Norway

3. Olafiaklinikken, Oslo University Hospital, Oslo, Norway

4. Division of Infectious Disease Control, Norwegian Institute of Public Health, Oslo, Norway

5. Faculty of Medicine, University of Oslo, Oslo, Norway

Unemo M, Golparian D, Syversen G, Vestrheim DF, Moi H. Two cases of verified clinical failures using internationally recommended first-line cefixime for gonorrhoea treatment, Norway, 2010. Euro Surveill. 2010;15(47):pii=19721. Available online: http://www.eurosurveillance.org/ViewArticle.aspx?Articleld=19721

Neisseria gonorrhoeae has developed resistance to most of the available therapeutic antimicrobials. The susceptibility to extended-spectrum cephalosporins, the last remaining first-line treatment option, is decreasing globally. This report describes the first two cases outside Japan of verified gonorrhoea clinical failures using internationally recommended firstline cefixime treatment. Enhanced awareness and more frequent follow-up examination, test-of-cure and appropriate verification/falsification of presumed clinical treatment failures, involving several clinical and laboratory parameters should be strongly emphasised worldwide.

\section{Introduction}

Gonorrhoea remains a public health problem worldwide. The prevalence of antimicrobial resistance (AMR) in Neisseria gonorrhoeae is high to all antimicrobials previously used as first-line treatments $[1,2]$. The susceptibility to the extended-spectrum cephalosporins (ESCs) ceftriaxone (injectable) and cefixime (oral), i.e. to the current internationally recommended first-line and last remaining treatment options, is decreasing worldwide $[1,2]$. Using ceftriaxone, no treatment failure has yet been reported. However, cefixime standard treatment (one oral dose of $400 \mathrm{mg}$ ) is the most common treatment in many countries due to the simple oral, single-dose regimen and its affordability. Verified treatment failures have so far only been reported in Japan [3]. Nevertheless in many cases, these failures may not be recognised because many of the gonorrhoea patients are also treated with azithromycin for suspected concomitant chlamydial infection $[1,4]$, and/ or because follow-up examinations and test-of-cure are rare [1]. In a recent review describing the latest World Health Organization (WHO) global initiatives to meet the public health challenge posed by the emergence of untreatable gonorrhoea, among others the ideal criteria for verification of gonorrhoea treatment failures are described. These involve several clinical and laboratory parameters [1].

This report describes the first two cases outside Japan of verified treatment failures of gonorrhoea using internationally recommended first-line treatment cefixime.

\section{Case reports \\ Case 1}

In July 2010 a Norwegian heterosexual man in his 305 presented to a hospital in Norway with purulent urethral discharge and dysuria (day 1). Urethritis and the presence of intracellular diplococci in polymorphonuclear lymphocytes were confirmed after methylene-blue staining of a urethral smear, and the patient was given one oral dose of cefixime $(400 \mathrm{mg})$. N. gonorrhoeae was also cultured from an additional urethral sample, and N. gonorrhoeae-specific DNA was detected in a urine sample using an in house PCR for the porA pseudogene [5]. On day 21, the patient returned with persisting symptoms, and microscopy, culture (urethral sample) and PCR (urine sample) remained positive for $N$. gonorrhoeae. The patient reported no sexual contacts between treatment and test-of-cure. He was then administered one dose of ceftriaxone $(500 \mathrm{mg}$ ) intramuscularly (IM). On day 29 , follow-up examination showed that the symptoms had resolved, and microscopy, culture (urethral sample), and PCR (urine sample) were negative for $N$. gonorrhoeae.

\section{Case 2}

In August 2010 another Norwegian heterosexual in his 30 s presented to the same hospital in Norway with similar symptoms and initial laboratory findings as case one and was treated with one oral dose of cefixime $(400 \mathrm{mg})$. On day 11 , he returned with persisting, although milder, symptoms. Microscopy, culture (urethral sample) and PCR (urine sample) were still positive for $N$. gonorrhoeae. The patient reported no sexual contacts between treatment and test-of-cure. He was 
administered one dose of ceftriaxone $500 \mathrm{mg}$ IM. On day 26, follow-up examination showed that the symptoms had resolved, and PCR (urine sample) was negative for N. gonorrhoeae.

\section{Characterisation of $N$. gonorrhoeae} isolates (before and after treatment)

All N. gonorrhoeae isolates were species-confirmed by sugar utilisation test, Phadebact Monoclonal GC Test (Pharmacia Diagnostics), and a porA pseudogene [5]. The characterisation of the isolates is summarised in the Table.

In both cases the pre- and post-treatment isolates were indistinguishable using full-length porB gene sequencing (identical sequence) and $N$. gonorrhoeae multiantigen sequence typing (NG-MAST; ST1407), performed as previously described [6]. Using Etest, the paired isolates displayed a cefixime minimum inhibitory concentration (MIC) of $0.5 \mathrm{mg} / \mathrm{L}$ and $0.25 / 0.5 \mathrm{mg} / \mathrm{L}$ in case 1 and 2, respectively, and indistinguishable antibiograms (beta-lactamase negative, ceftriaxone: $0.125 \mathrm{mg} / \mathrm{L}$, ciprofloxacin: $>32 \mathrm{mg} / \mathrm{L}$, azithromycin: $0.5 \mathrm{mg} / \mathrm{L}$, spectinomycin: $12 \mathrm{mg} / \mathrm{L}$, and ampicillin: $1-4 \mathrm{mg} / \mathrm{L})$. According to the breakpoints stated by the Committee on Antimicrobial Susceptibility Testing (EUCAST), these isolates were considered resistant to cefixime (MIC>0.12 mg/L). Sequencing of resistance determinants for ESCs (penA, mtrR and porBib alterations) was performed as previously described [7]. All four isolates from the two cases contained an identical penA mosaic allele, which had previously been correlated to treatment failures in Japan, and $m \operatorname{tr} R$ and pen $B$ resistance determinants that enhance the MICs for ESC further (Table) $[8,9]$.

\section{Discussion and conclusion}

This report describes the first two cases outside Japan - with no known links to Japan - of verified clinical treatment failures of urogenital gonorrhoea using standard first-line cefixime treatment, i.e. one dose of $400 \mathrm{mg}$. The treatment failures were strictly verified using several clinical and laboratory parameters in full accordance to WHO criteria $[1,10]$, i.e. a detailed clinical history was recorded, re-exposure and reinfection was ruled out, the pre-treatment and post-treatment isolates were phenotypically and genetically indistinguishable by highly discriminatory molecular epidemiological typing methods, the MICs of cefixime were substantially enhanced (in vitro-resistance according to current breakpoints), and the isolates contained genetic resistance determinants causing the enhanced cefixime MICs. According to Monte Carlo simulations, a $400 \mathrm{mg}$ dose of cefixime results in median times of free cefixime above the MIC ( $\left.f \mathrm{~T}_{\text {MIC }}\right)$ of only $14.9 \mathrm{~h}$ (12.318.3 h) and 11.4 h (9.0-14.2 h) for the detected MICs of $0.25 \mathrm{mg} / \mathrm{L}$ and $0.5 \mathrm{mg} / \mathrm{L}$, respectively [4]. Accordingly, these levels of cefixime MICs are high enough to cause treatment failures. Most worrying, the gonococcal isolates from the present treatment failures were identified as ST1407, which is a multi-resistant strain that, together with its evolving subtypes, has been shown to circulate in many countries worldwide [11].

In conclusion, cases of clinical failures of urogenital gonorrhoea using the internationally recommended first-line treatment cefixime have now also occurred outside Japan. Besides the two cases identified in Norway (with places of exposure in the Philippines and Spain/Norway), similar rare cases are likely to be identified elsewhere. Accordingly, an enhanced awareness of the existence of failures using cefixime for gonorrhoea treatment needs to be strongly emphasised worldwide as well as the need for more frequent follow-up examination, test-of-cure and appropriate verification/falsification of presumed clinical treatment

TABLE

Characteristics of two Neisseria gonorrhoeae cases of verified clinical failure using internationally recommended first-line cefixime treatment, Norway, 2010

\begin{tabular}{|c|c|c|c|c|c|c|c|c|c|c|}
\hline \multirow{2}{*}{$\begin{array}{l}\text { Age (years) } \\
\text { / Sex }\end{array}$} & \multirow{2}{*}{$\begin{array}{c}\text { Possible place } \\
\text { of exposure }\end{array}$} & \multirow{2}{*}{ Treatment } & \multirow{2}{*}{$\begin{array}{l}\text { Diagnostics } \\
\text { (pre-treatment/ } \\
\text { post-treatment } \\
\text { isolate) }\end{array}$} & \multicolumn{2}{|c|}{$\mathrm{MIC}(\mathrm{mg} / \mathrm{L})$} & \multirow[t]{2}{*}{ NG-MAST } & \multirow[t]{2}{*}{$\begin{array}{l}\operatorname{pen} A \\
\text { allele }^{a}\end{array}$} & \multirow[t]{2}{*}{$m t^{2} R^{b}$} & \multicolumn{2}{|c|}{$\operatorname{pen}^{c}$} \\
\hline & & & & Cefixime & Ceftriaxone & & & & & \\
\hline \multirow{2}{*}{$37 /$ male } & \multirow{2}{*}{ Philippines } & \multirow{2}{*}{$\begin{array}{l}\text { Cefixime } \\
400 \mathrm{mg}^{\prime} 1 \\
\text { oral dose }\end{array}$} & $\begin{array}{l}\text { Microscopy, } \\
\text { culture, PCR }\end{array}$ & 0.5 & 0.125 & ST1407 & Mosaic & $\begin{array}{l}\text { A-del in } \\
\text { promoter }\end{array}$ & G120K & $\mathrm{A} 121 \mathrm{~N}$ \\
\hline & & & $\begin{array}{l}\text { Microscopy, } \\
\text { culture, PCR }\end{array}$ & 0.5 & 0.125 & $\mathrm{ST} 1407$ & Mosaic & $\begin{array}{l}\text { A-del in } \\
\text { promoter }\end{array}$ & G120K & $\mathrm{A} 121 \mathrm{~N}$ \\
\hline \multirow{2}{*}{$31 /$ male } & \multirow{2}{*}{$\begin{array}{l}\text { Spain/ } \\
\text { Norway }\end{array}$} & \multirow{2}{*}{$\begin{array}{l}\text { Cefixime } \\
400 \mathrm{mg}^{\prime} 1 \\
\text { oral dose }\end{array}$} & $\begin{array}{l}\text { Microscopy, } \\
\text { culture, PCR }\end{array}$ & 0.25 & 0.125 & ST1407 & Mosaic & $\begin{array}{l}\text { A-del in } \\
\text { promoter }\end{array}$ & G120K & $\mathrm{A} 121 \mathrm{~N}$ \\
\hline & & & $\begin{array}{l}\text { Microscopy, } \\
\text { culture, PCR }\end{array}$ & 0.5 & 0.125 & ST1407 & Mosaic & $\begin{array}{l}\text { A-del in } \\
\text { promoter }\end{array}$ & G120K & $\mathrm{A} 121 \mathrm{~N}$ \\
\hline
\end{tabular}

MIC: minimum inhibitory concentration; NG-MAST: Neisseria gonorrhoeae multiantigen sequence typing; PCR: polymerase chain reaction; ST: sequence type.

a penA mosaic allele encodes the mosaic penicillin-binding protein 2 (PBP2), which causes decreased susceptibility to extended-spectrum cephalosporins.

${ }^{b} \mathrm{~A}$-del in promoter: characteristic single nucleotide $(A)$ deletion in the inverted repeat of the promoter region of mtrR that causes overexpression of the MtrCDE efflux pump, which results in a further decreased susceptibility to extended-spectrum cephalosporins.

c penB: alterations of amino acids 120 and 121 in the porin PorB1b that cause a decreased intake of extended-spectrum cephalosporins and, accordingly, a further decreased susceptibility to extended-spectrum cephalosporins. 
failures. Pharyngeal gonorrhoea poses an additional challenge as it is often asymptomatic, more difficult to eradicate, and may act as a reservoir for emergence of resistance [1]. Accordingly, it is important to collect information regarding the patients' sexual practices and to take also extragenital samples.

\section{References}

1. Tapsall JW, Ndowa F, Lewis DA, Unemo M. Meeting the public health challenge of multidrug- and extensively drugresistant Neisseria gonorrhoeae. Expert Rev Anti Infect Ther. 2009;7(7):821-34.

2. Barry PM, Klausner JD. The use of cephalosporins for gonorrhea: the impending problem of resistance. Expert Opin Pharmacother. 2009;10(4):555-77.

3. Yokoi S, Deguchi T, Ozawa T, Yasuda M, Ito S, Kubota Y, et al. Threat to cefixime treatment of gonorrhea. Emerg Infect Dis. 2007;13(8):1275-7.

4. Chisholm SA, Mouton JW, Lewis DA, Nichols T, Ison CA, Livermore DM. Cephalosporin MIC creep among gonococci: time for a pharmacodynamic rethink? J Antimicrob Chemother. 2010;65(10):2141-8.

5. Hjelmevoll SO, Olsen ME, Sollid JU, Haaheim H, Unemo $\mathrm{M}$, Skogen V. A fast real-time polymerase chain reaction method for sensitive and specific detection of the Neisseria gonorrhoeae porA pseudogene. J Mol Diagn. 2006;8(5):574-81.

6. Unemo M, Sjöstrand A, Akhras M, Gharizadeh B, Lindbäck E, Pourmand N, et al. Molecular characterization of Neisseria gonorrhoeae identifies transmission and resistance of one ciprofloxacin-resistant strain. APMIS. 2007;115(3):231-41.

7. Unemo M, Fasth O, Fredlund H, Limnios A, Tapsall J. Phenotypic and genetic characterization of the 2008 WHO Neisseria gonorrhoeae reference strain panel intended for global quality assurance and quality control of gonococcal antimicrobial resistance surveillance for public health purposes. J Antimicrob Chemother. 2009;63(6):1142-51.

8. Lindberg R, Fredlund H, Nicholas R, Unemo M. Neisseria gonorrhoeae isolates with reduced susceptibility to cefixime and ceftriaxone: Association with genetic polymorphisms in penA, mtrR, porB1b, and ponA. Antimicrob Agents Chemother. 2007;51(6):2117-22.

9. Zhao S, Duncan M, Tomberg J, Davies C, Unemo M, Nicholas R. Genetics of chromosomally mediated intermediate resistance to ceftriaxone and cefixime in Neisseria gonorrhoeae. Antimicrob Agents Chemother. 2009;53(9):3744-51.

10. WHO GASP Document. Rationale and applications for the current (2008) WHO panel of Neisseria gonorrhoeae for antimicrobial resistance surveillance for public health purposes, and instructions for their use. In: Technical document Do07-0408-1\#1 (2008) Sydney: WHO Collaborating Centre for STD.

11. Golparian D, Hellmark B, Fredlund H, Unemo M. Emergence, spread and characteristics of Neisseria gonorrhoeae isolates with in vitro decreased susceptibility and resistance to extended-spectrum cephalosporins in Sweden. Sex Transm Infect. 2010;86(6):454-60. 